\title{
Anthrovision
}

Vaneasa Online Journal

$1.2 \mid 2013$

Varia

\section{Histoires de fantômes pour grandes personnes}

Exposition de Georges Didi-Huberman et Arno Gisinger, Le Fresnoy, Studio national des arts contemporains 2012

Nadine Wanono

\section{(2) OpenEdition}

Journals

Édition électronique

URL : http://journals.openedition.org/anthrovision/819

DOI : 10.4000/anthrovision.819

ISSN : 2198-6754

Éditeur

VANEASA - Visual Anthropology Network of European Association of Social Anthropologists

Référence électronique

Nadine Wanono, « Histoires de fantômes pour grandes personnes », Anthrovision [En ligne], 1.2 | 2013, mis en ligne le 01 août 2013, consulté le 22 septembre 2020. URL : http://journals.openedition.org/ anthrovision/819; DOI : https://doi.org/10.4000/anthrovision.819

Ce document a été généré automatiquement le 22 septembre 2020

(c) Anthrovision 


\title{
Histoires de fantômes pour grandes personnes
}

\author{
Exposition de Georges Didi-Huberman et Arno Gisinger, Le Fresnoy, \\ Studio national des arts contemporains 2012
}

\section{Nadine Wanono}

1 Sur l'invitation d'Alain Flisher, directeur du Studio national des arts contemporains Le Fresnoy, Georges Didi-Huberman a conçu une planche intitulée Mnemosyne 42 en hommage au travail d'Aby Warburg, historien de l'art. Dans les dernières années de sa vie (1924-1929), Aby Warburg met en scène des planches composées d'images, de photographies, de reproduction afin de rendre visible ce qu'il appelait «la survivance ».

2 En 2002, Georges Didi-Huberman consacre à ce thème central un ouvrage intitulé l'image survivante, histoire de l'art et temps des fantômes, où il présente ce concept de Nachleben, autour duquel Warburg va construire son oeuvre Mnemosyne. Georges DidiHuberman présente les sources d'inspirations et de réflexions du célèbre historien de l'art qui se consacre à la mise en évidence de la paradoxale vie des images, leur capacité de « revenance » de hantise, leur nature de fantôme. Nous pouvons citer Georges DidiHuberman lorsqu'il rappelle que «Benjamin a posé qu'une histoire de la culture ne va pas sans la mise au jour d'un inconscient de la vision. Aby Warburg avait compris qu'une telle mise au jour n'est possible qu'à interroger cet “inconscient du temps" qu'est la survivance. »

3 Pour Alain Fisher, il s'agissait d'investir l'espace de la grande nef du Fresnoy (mille mètres carrés environ) afin que tout soit visible depuis la coursive du premier étage où, seraient installées les photos de l'exposition de Atlas, suite, séries d'images d'Arno Gisinger réalisées à partir de l'exposition Atlas (Didi-Huberman et Gisinger 2013).

4 A partir de la quarante-deuxième planche de Mnémosyne, consacrée au motif de la Pietà et aux lamentations que les vivants produisent, chantent, hurlent ou murmurent devant leurs morts, Georges Didi-Huberman a conçu un mur d'images fixes ou en mouvement posé au sol, que le spectateur découvre comme depuis le pont d'un bateau, accoudé sur le bastingage. Avec la même dynamique que Warburg, Georges Didi- 
Huberman entrelace les souvenirs construits à partir de films cultes (Pasolini, Eisenstein, Dreyer) ou de films plus contemporains (Godard, Paradjanov, Harun Farocki, Zhao Liang ou encore Jean Rouch) qui renvoient indistinctement à des souvenirs, émotions, époques de vie, moments historiques, où le spectateur est pris dans cet entrelacs de liens tissés au creux de sa mémoire et de sa personnalité en complète évolution. Le spectateur est projeté dans le vide de cet espace qui s'ouvre devant lui, le met perpendiculaire aux images qu'il découvre comme au fond d'une piscine où nous serions invités à accomplir un travail d'excavation archéologique. La faible lumière ambiante, le son qui apparaît de manière aléatoire renforce chez le spectateur cette fragilité des souvenirs qui apparaissent et se superposent. Georges Didi-Huberman parle de feuilletage de la mémoire, qui se construit du passé pour impressionner notre futur. Les fantômes nous accompagnent dans la traversée du temps, ceux que nous connaissons, ceux qui nous restent à identifier ou à inventer.

Cette exposition démonstration rappelait aux visiteurs le rôle essentiel de l'imagination dans le processus de connaissances et donnait aux spectateurs un plaisir infini à l'expérimenter, à le concevoir et à l'élaborer en toute liberté dans cet espace magique du studio Le Fresnoy.

Capture des écrans des extraits de films



Photo prise lors de la visite de l'exposition. G.Gauthier. 


\section{AUTEUR}

NADINE WANONO

Centre d'études des mondes africains, CNRS, Paris I, EPHE 